Vol.01/ No. 02

Pages: 91- 101

https://www.irojournals.com/iroiip/

DOI: https://doi.org/10.36548/jiip.2019.2.004

\title{
BIOMEDICAL IMAGE ANALYSIS USING SEMANTIC SEGMENTATION
}

\author{
Dr. Bindhu V, \\ Professor and Head, Department of Electronics and Communication Engineering, \\ PPG Institute of Technology, \\ Villankurichi Saravanampatti P.O, \\ Coimbatore, Tamil Nadu, India. \\ Email id: vbindhuppg@ieee.org
}

\begin{abstract}
Semantic Segmentation is a very active area of research in the examining the medical images. The failure in the conventional segmentation methods to preserve the full resolution throughout the network led to the research's that developed methods to protect the resolution of the images. The proposed method involves the semantic segmentation model for the biomedical images by utilizing the encoder/decoder structure to down sample the spatial resolution of the input data and develop a lower resolution feature mapping that are very effective at distinguishing between the classes and then perform the up samples to have a full-resolution segmentation map of the biomedical images reducing the diagnostic time. The frame work put forth utilizes a pixel to pixel fully trained cascaded convolutional neural network for the task of image segmentation. The evaluation biomedical image analysis using the semantic segmentation shows the performance improvement achieved by the minimization of the time required in testing and the augmentation in the analysis performed by the radiologist.
\end{abstract}

Keywords: Fully Connected Convolutional Networks, Biomedical Images, Down Sampling, Up Sampling, Encoder/Decoder

\section{INTRODUCTION}

The latest developments on the machine learning technology including the deep learning approaches have gained a huge attraction in current researches [1]. The deep learning process utilizes the efficient algorithm to identify as well as learn from the images that were fed as raw data to distinguish the objects between different classes. The evolution of the semantic segmentation started with the emergence of the computer vision applications [2] that necessitated the identification of the basic component such as the lines, curves or the gradients of the image, this provided only outward information of the images excluding the comprehensive particulars of the image [3]. This was made possible by the evolution of the full pixel semantic segmentation that provided the understanding of the image from the pixel level. There are two other methods along with the semantic segmentation that support in identifying or clustering the pixels together by allocating identification tags for each class. The figure.1 shows the processes in identifying each pixel or the grouping pixels [4-5].

ISSN: 2582- 4252 
Vol.01/ No. 02

Pages: 91- 101

https://www.irojournals.com/iroiip/

DOI: https://doi.org/10.36548/jiip.2019.2.004

\section{Image classification}

- Identifies the objects present in the image

\section{Object Recognition and detection}

- Identifies the object present in the image and where (through the bounding box)

\section{Semantic Segmentation}

- Identifying the object present in the image and where (by finding all pixels that belongs to it)

Fig.1 Process in Identifying the Pixels and Grouping Pixels

As the early computer vision problems enabled only to identify the edges, the lines, curves or the gradients, they hardly provided the image insights at pixel level, or in the way the human sees it [6-7]. The semantic segmentation provides the pixel level information of the images by taking in the raw data that is the two dimensional images as the input and transforming them into a mask with the highlighted region of interest, it is often referred as the full pixel semantic segmentation (FPSS), as the each pixel in the image is assigned with an identification tag depending on the objects of interest (OBI).

It clusters the parts of images belonging to the same class to identify solutions to the problem for the numerous of applications that were associated with the computer vision. The semantic segmentation shows much difference in processing an image compared to the other image based tasks listed in the fig .1; it even aids the machine learning model to gain knowledge about the every single pixel of the image [9-11].

This image segmentation process is broadly classified into two types as

ISSN: 2582- 4252 
Journal of Innovative Image Processing (JIIP) (2019)

Vol.01/ No. 02

Pages: 91- 101

https://www.irojournals.com/iroiip/

DOI: https://doi.org/10.36548/jiip.2019.2.004

i. Standard Semantic Segmentation

ii. Instance-aware semantic segmentation

The table. 1 below provides the types, description and the representation of the semantic segmentation.

\begin{tabular}{|l|l|l|}
\hline \multicolumn{1}{|c|}{ Types } & \multicolumn{1}{|c|}{ Description } & \multicolumn{2}{|c|}{ Segmentation } \\
\hline Standard semantic & $\begin{array}{l}\text { It is called the full } \\
\text { Sixel semantic } \\
\text { segmentation } \\
\text { It labels each pixel } \\
\text { of an image with the } \\
\text { corresponding class } \\
\text { of what is being } \\
\text { represented. }\end{array}$ & $\begin{array}{l}\text { It is a subtype of } \\
\text { semantic } \\
\text { segmentation } \\
\text { It classifies each } \\
\text { pixel as an object } \\
\text { class and separates } \\
\text { objects of the same } \\
\text { class }\end{array}$ \\
\hline semantic segmentation
\end{tabular}

Table.2 Types of Semantic Segmentation

The segmentation process aims in identifying the region of the images and labeling the meaning indicated by that pixel for each pixel rather than finding out the meaning of the entire image or the partial image. There are many conventional segmentation approaches to gain the meaningful information's from the images by directly learning the mapping from the raw data fed in and the performing the segmentation process, they were quite costly as well as computationally expensive.

ISSN: 2582- 4252

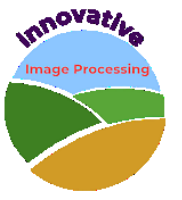


Journal of Innovative Image Processing (JIIP) (2019)

Vol.01/ No. 02

Pages: 91- 101

https://www.irojournals.com/iroiip/

DOI: https://doi.org/10.36548/jiip.2019.2.004

So the paper puts forward the encoder/decoder structure to down sample the spatial resolutions of the input, to develop a lower resolution feature mapping that are highly effective in discriminating between the classes and up samples the feature representation into a full resolution segmentation map of the biomedical data set (kaggle lung CT dataset and the UCSB) as the machines can boost the examination performed by the radiologist minimizing the time required to run the diagnostics test.

\section{RELATED WORKS}

Ravi Shankar, Hariharan, et al [12] the author provides the segmentation model for the problem of the ultrasound kidney segmentation by utilizing the U-NET fully convolutional networks. Christ, Patrick et al [13] in his paper details the "Automatic liver and lesion segmentation in CT using cascaded fully convolutional neural networks and 3D conditional random fields." Drozdzal, et al [14] the author details "the importance of skip connections in biomedical image segmentation and its utilization in recover the spatial information's that were lost during the down sampling"

Milletari, Fausto et al [15] the author proposes the fully convolutional neural network, V-net in segmenting a high volume of medical images. Yi, Zhao, et al [16] proposes the "novel algorithm for the automatic segmentation and the classification of the brain tissues from 3D MR scans" Shvets, Alexey et al [17] provides the "Automatic instrument segmentation in robot-assisted surgery using deep learning."

Kainz, Philipp et al [18] presents the "Semantic segmentation of colon glands with deep convolutional neural networks and total variation segmentation." Lu, Le, et al [19] details the performance enhancement in the medical image computing by integrating the deep learning and the CNN. Ronneberger, et al [20] the author details the utilization of the u-net architectures in segmenting the biomedical images.

\section{PROPOSED WORK}

The section presents the construction of the convolutional neural network model to analyze the biomedical images, the segmentation frame work included a cascade fully connected convolutional neural network to encoder /decoder, skip connection and segments the images respectively. The frame work utilizes the V-net architecture [15] to

ISSN: 2582- 4252 
Journal of Innovative Image Processing (JIIP) (2019)

Vol.01/ No. 02

Pages: 91- 101

https://www.irojournals.com/iroiip/

DOI: https://doi.org/10.36548/jiip.2019.2.004

examine the biomedical images. The frame work in the fig. 2 provides the overview of the segmentation model in analyzing the biomedical images.

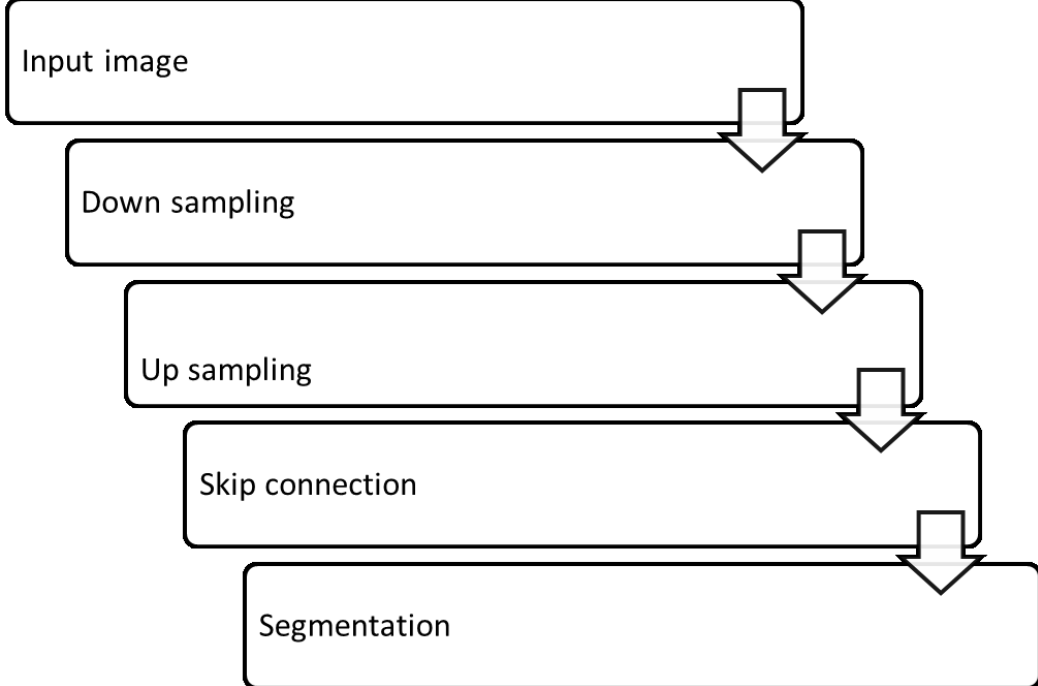

Fig.2.Over View of the Semantic Segmentation

The image fed as the raw data to the frame work is initially down sampled using the pooling operations, reducing the spatial resolution of the input in order to produce a low resolution feature mapping to be learnt and further a learned up-sampling is applied using the transpose convolutions. Where a single value from the low resolution feature map is taken and multiplied with the weights in the filter using the single value, producing the weighted values at the output. The figure. 3 below shows the up sampling process

ISSN: 2582- 4252 
Journal of Innovative Image Processing (JIIP) (2019)

Vol.01/ No. 02

Pages: 91- 101

https://www.irojournals.com/iroiip/

DOI: https://doi.org/10.36548/jiip.2019.2.004

\section{Output}

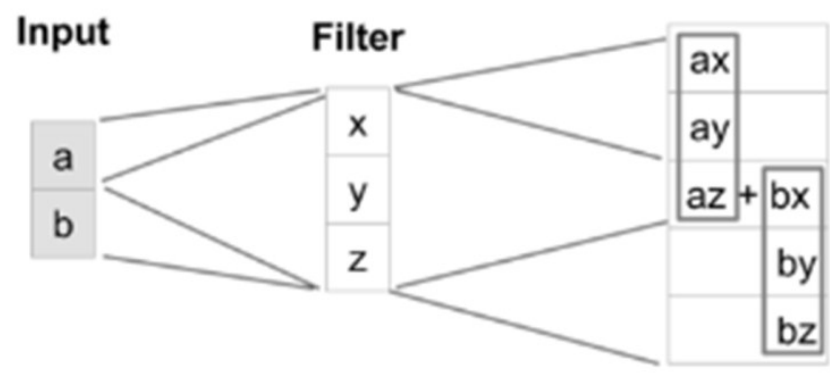

Fig.3 Up Sampling through Transpose Convolution

The proposed method utilizes the AlexNet as the encoder module and the affixes the decoder module with transpose convolutional layers to up sample the feature maps to the full -resolution segmentation map. The system is trained based on the pixel wise entropy; however as the decoder module struggles to produce a fine grained segmentation the skip connection [14] process in involved in the network in the earlier layers to deliver the necessary particulars required in the reconstruction of the accurate shapes for the segmentation boundaries an reproduce a fine grained segmentation The process is proposed in the form of V-net architecture[15] to expand the path and provide a very concise localization. The fig. 4 below shows the V- net architecture to segment the biomedical images.
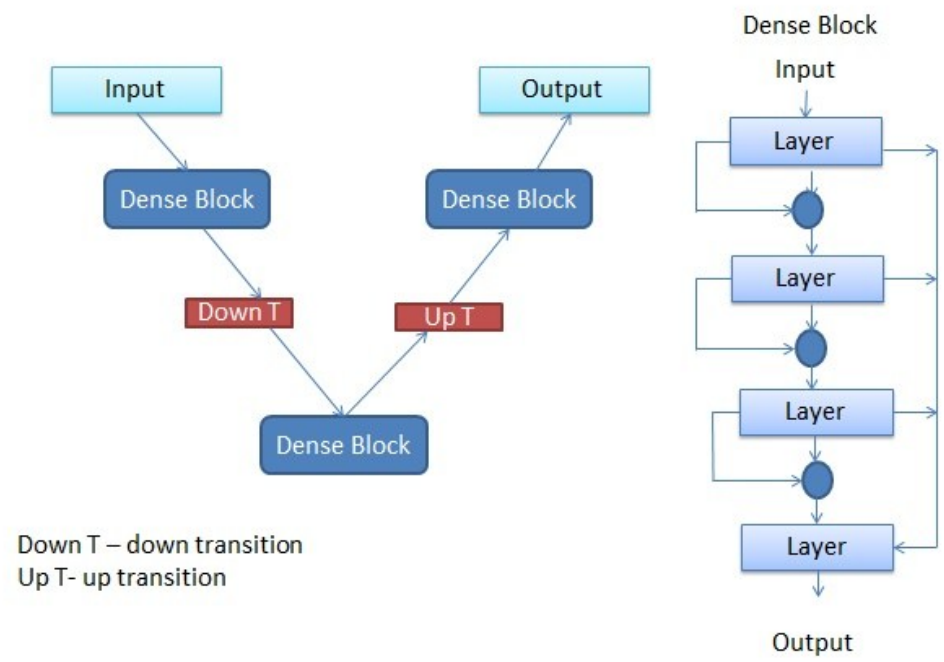

ISSN: 2582- 4252 
Journal of Innovative Image Processing (JIIP) (2019)

Vol.01/ No. 02

Pages: 91- 101

https://www.irojournals.com/iroiip/

DOI: https://doi.org/10.36548/jiip.2019.2.004

Fig.4 V-net architecture

\section{RESULTS}

The evaluation includes the biomedical data set (kaggle lung CT dataset and the UCSB) to train as well as validate. The experiment includes metrics such as the binary cross entropy (BCE), dice coefficient (DC) and the intersection over union IOU . The table.2 below presents the details of the metrics used.

\begin{tabular}{|c|c|}
\hline Metrics & \multicolumn{1}{|c|}{ Description } \\
\hline Binary cross entropy & $\begin{array}{l}\text { A common metric and loss } \\
\text { function for binary } \\
\text { classification for measuring the } \\
\text { probability of misclassification }\end{array}$ \\
\hline Dice coefficient & $\frac{2(\text { area overlapped })}{\text { total area }}$ \\
\hline Intersection over union & $\frac{\text { area overlapped }}{\text { overall area }}$ \\
\hline
\end{tabular}

Table.2 Metric Used

The segmentation network is trained with 75 epochs utilizing the Adam optimizer at the learning rate of $1-e^{4}$ and the step LR with the $.1 \gamma$ for every 15 epochs. The loss function is the combination of the BCE and the DC the fig 5(a) shows the percentage of BCE, DC and IOU in validation and 5(b) shows the accuracy percentage in training and validation.

ISSN: 2582- 4252 
Journal of Innovative Image Processing (JIIP) (2019)

Vol.01/ No. 02

Pages: 91- 101

https://www.irojournals.com/iroiip/

DOI: https://doi.org/10.36548/jiip.2019.2.004

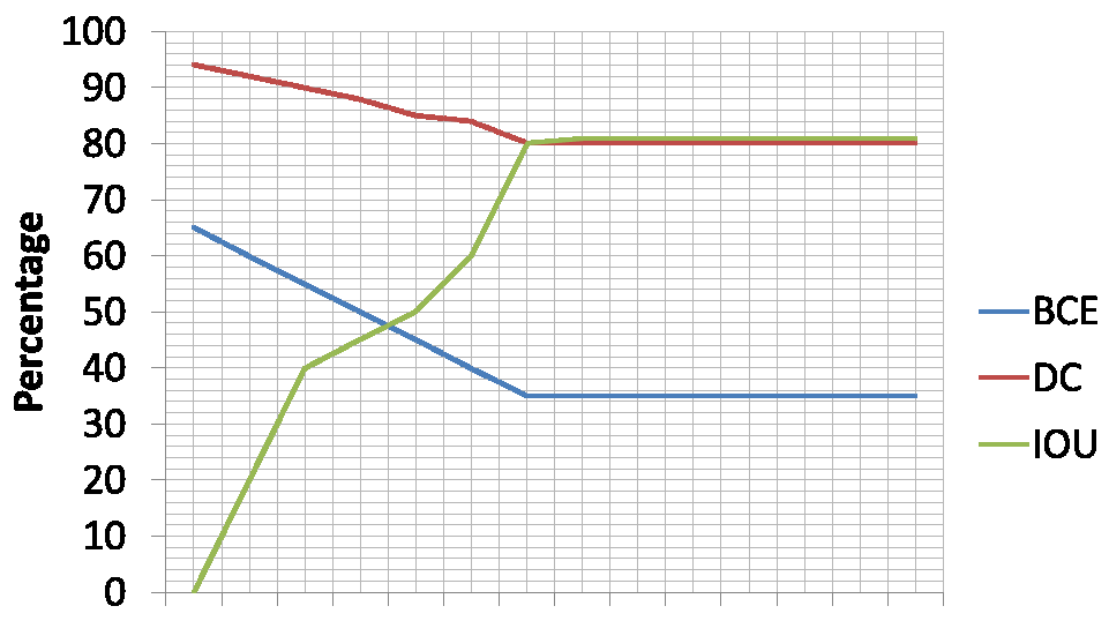

1015202530354045505560657075

Number of Epochs

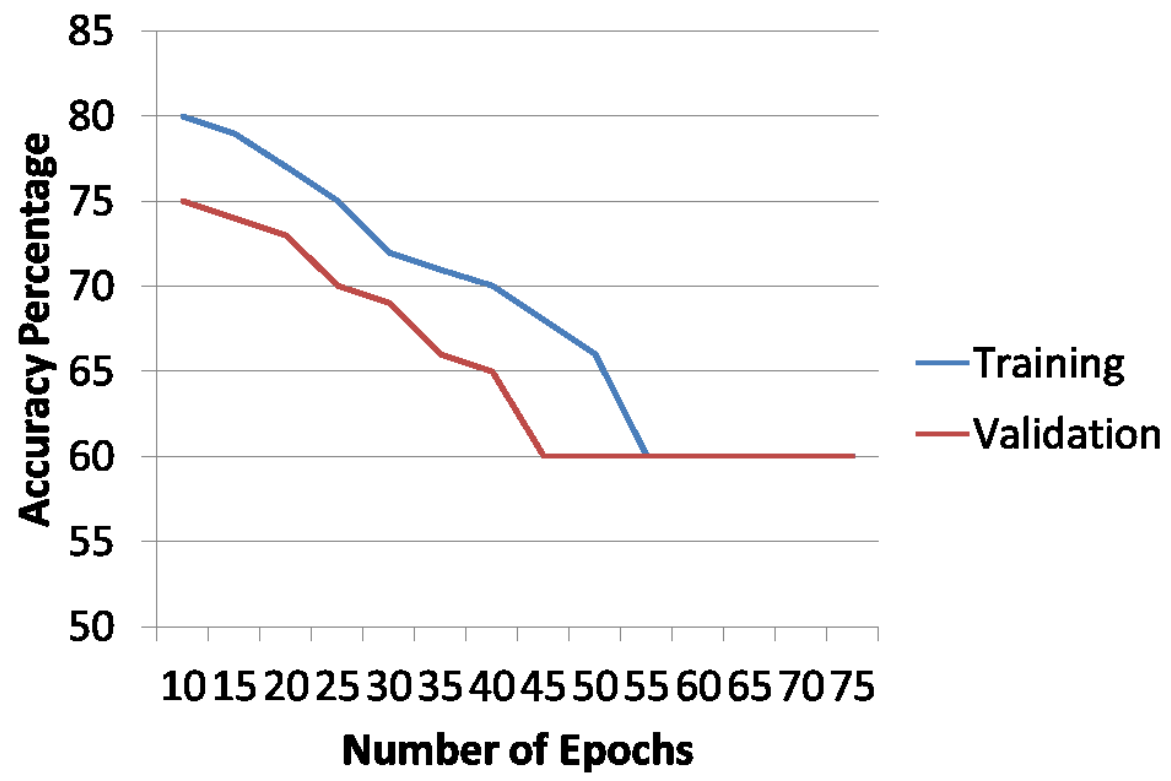

Fig.5 (a) BCE, DC and IOU and 5. (b) Accuracy Percentage

ISSN: 2582- 4252 
Journal of Innovative Image Processing (JIIP) (2019)

Vol.01/ No. 02

Pages: 91- 101

https://www.irojournals.com/iroiip/

DOI: https://doi.org/10.36548/jiip.2019.2.004

\section{CONCLUSION}

The V-net architecture cascade with the AlexNet as the encoder module and the transpose convolution as the decoder module applied for the biomedical segmentation produces a very high performance with the rational amount of training epochs at a rational training time. in future the paper is to put forth the capsule network for the semantic segmentation process to elude the cost and the time spent in regaining the resolution lost in the pooling.

\section{References}

[1] Raj, Jennifer S. "A COMPREHENSIVE SURVEY ON THE COMPUTATIONAL INTELLIGENCE TECHNIQUES AND ITS APPLICATIONS." Journal of ISMAC 1, no. 03 (2019): 147-159.

[2] Joseph, S. Iwin Thanakumar. "SURVEY OF DATA MINING ALGORITHM'S FOR INTELLIGENT COMPUTING SYSTEM." Journal of trends in Computer Science and Smart technology (TCSST) 1, no. 01 (2019): 14-24.

[3] Raj, Jennifer S., and J. Vijitha Ananthi. "RECURRENT NEURAL NETWORKS AND NONLINEAR PREDICTION IN SUPPORT VECTOR MACHINES." Journal of Soft Computing Paradigm (JSCP) 1, no. 01 (2019): 33-40.

[4] Sathesh, A. "PERFORMANCE ANALYSIS OF GRANULAR COMPUTING MODEL IN SOFT COMPUTING PARADIGM FOR MONITORING OF FETAL ECHOCARDIOGRAPHY." Journal of Soft Computing Paradigm (JSCP) 1, no. 01 (2019): 14-23.

[5] Smys, S., and Jennifer S. Raj. "VIRTUAL REALITY SIMULATION AS THERAPY FOR POSTTRAUMATIC STRESS DISORDER (PTSD)." Journal of Electronics 1, no. 01 (2019): 24-34.

[6] Vijayakumar, T. "COMPARATIVE STUDY OF CAPSULE NEURAL NETWORK IN VARIOUS APPLICATIONS." Journal of Artificial Intelligence 1, no. 01 (2019): 19-27.

[7] Bashar, Abul. "SURVEY ON EVOLVING DEEP LEARNING NEURAL NETWORK ARCHITECTURES." Journal of Artificial Intelligence 1, no. 02 (2019): 73-82.

[8] Manoharan, Samuel. "AN IMPROVED SAFETY ALGORITHM FOR ARTIFICIAL INTELLIGENCE ENABLED PROCESSORS IN SELF DRIVING CARS." Journal of Artificial Intelligence 1, no. 02 (2019): 95-104.

[9] Koresh, Mr H. James Deva. "COMPUTER VISION BASED TRAFFIC SIGN SENSING FOR SMART TRANSPORT." Journal of Innovative Image Processing (JIIP) 1, no. 01 (2019): 11-19.

ISSN: 2582- 4252 
Journal of Innovative Image Processing (JIIP) (2019)

Vol.01/ No. 02

Pages: 91- 101

https://www.irojournals.com/iroiip/

DOI: https://doi.org/10.36548/jiip.2019.2.004

[10] Chandy, Abraham. "A REVIEW ON IOT BASED MEDICAL IMAGING TECHNOLOGY FOR HEALTHCARE APPLICATIONS." Journal of Innovative Image Processing (JIIP) 1, no. 01 (2019): 51-60.

[11] Smys, S. "VIRTUAL REALITY GAMING TECHNOLOGY FOR MENTAL STIMULATION AND THERAPY." Journal of Information Technology 1, no. 01 (2019): 19-26.

[12] Ravishankar, Hariharan, Rahul Venkataramani, Sheshadri Thiruvenkadam, Prasad Sudhakar, and Vivek Vaidya. "Learning and incorporating shape models for semantic segmentation." In International Conference on Medical Image Computing and Computer-Assisted Intervention, pp. 203-211. Springer, Cham, 2017.

[13] Christ, Patrick Ferdinand, Mohamed Ezzeldin A. Elshaer, Florian Ettlinger, Sunil Tatavarty, Marc Bickel, Patrick Bilic, Markus Rempfler et al. "Automatic liver and lesion segmentation in CT using cascaded fully convolutional neural networks and 3D conditional random fields." In International Conference on Medical Image Computing and Computer-Assisted Intervention, pp. 415-423. Springer, Cham, 2016.

[14] Drozdzal, Michal, Eugene Vorontsov, Gabriel Chartrand, Samuel Kadoury, and Chris Pal. "The importance of skip connections in biomedical image segmentation." In Deep Learning and Data Labeling for Medical Applications, pp. 179-187. Springer, Cham, 2016.

[15] Milletari, Fausto, Nassir Navab, and Seyed-Ahmad Ahmadi. "V-net: Fully convolutional neural networks for volumetric medical image segmentation." In 2016 Fourth International Conference on $3 D$ Vision (3DV), pp. 565-571. IEEE, 2016.

[16] Yi, Zhao, Antonio Criminisi, Jamie Shotton, and Andrew Blake. "Discriminative, semantic segmentation of brain tissue in MR images." In International Conference on Medical Image Computing and Computer-Assisted Intervention, pp. 558-565. Springer, Berlin, Heidelberg, 2009.

[17] Shvets, Alexey A., Alexander Rakhlin, Alexandr A. Kalinin, and Vladimir I. Iglovikov. "Automatic instrument segmentation in robot-assisted surgery using deep learning." In 2018 17th IEEE International Conference on Machine Learning and Applications (ICMLA), pp. 624-628. IEEE, 2018

[18] Kainz, Philipp, Michael Pfeiffer, and Martin Urschler. "Semantic segmentation of colon glands with deep convolutional neural networks and total variation segmentation." arXiv preprint arXiv:1511.06919(2015).

[19] Lu, Le, Yefeng Zheng, Gustavo Carneiro, and Lin Yang. "Deep learning and convolutional neural networks for medical image computing." Advances in Computer Vision and Pattern Recognition; Springer: New York, NY, USA (2017).

ISSN: 2582- 4252 
Journal of Innovative Image Processing (JIIP) (2019)

Vol.01/ No. 02

Pages: 91- 101

https://www.irojournals.com/iroiip/

DOI: https://doi.org/10.36548/jiip.2019.2.004

[20] Ronneberger, Olaf, Philipp Fischer, and Thomas Brox. "U-net: Convolutional networks for biomedical image segmentation." In International Conference on Medical image computing and computer-assisted intervention, pp. 234-241. Springer, Cham, 2015.

ISSN: 2582- 4252 